modes of imaging.

The early days of the evolution of nuclear medicine imaging are completely missed out, and the book says that the first computerized image was an X-ray computed tomography one, but it was actually from a gamma camera. The social side is well covered, but is a bit obsessed with violence and the assassinations of US presidents, as these are a means of illustrating the use, or not, of the appropriate imaging mode of the time.

The book is clearly intended for readers in the United States. Whether the US chauvinism is deliberate or not, the effect is the same: the playing down of UK and European contributions. Nature urges book reviewers to avoid parochialisms as they can be irritating to readers in other countries; I found the US parochialisms in this book downright annoying. The British contributions to nuclear medicine, positron emission tomography, ultrasound and particularly to magnetic resonance imaging are largely ignored. Of the 46 people Kevles interviewed for the book, only two were UK scientists. The index references 21 US institutions but only two British and one German. The first radiological society in the world was founded in 1897 in Britain, but is not even mentioned. It seems that history is being rewritten by journalists; perhaps history is always like this. Can one really rely on what it says in the history books? Once one author gets it wrong, then it may become perpetuated in books that follow. There ought to be more history books written by the scientists themselves.

Examples of this chauvinism are legion. In the chapter on ultrasound I could find no mention of the Nuclear Enterprises Diasonograph, which was one of the leading commercial instruments for many years; Ian Donald's pioneering work in Glasgow is not mentioned until right at the end, and then somewhat grudgingly; it says that in 1980 magnetic resonance imaging was not clinically useful, whereas British National Health Service patients in Aberdeen were being usefully imaged from August 1980 onwards.

I must declare an interest here as I have devoted much of my working life to developing medical imaging, particularly in nuclear medicine and magnetic resonance imaging. I therefore find it rather galling that most of it was completely ignored; that, when I was mentioned, I was described as a medical physician, when I am, in fact, a physicist; and that my major contribution to magnetic res- onance imaging - the team breakthrough that made it useful clinically - was credited to others in the United States! Neither is credit given to Ian Young's team in London for making the first superconducting magnet imager work at Hammersmith Hospital in 1981. There is also no mention of Japanese work until the chapter on ultrasound, and no mention that for years Japan was second only to the United States in its take-up of magnetic resonance imaging. Perhaps this book's subtitle should have been "Medical Imaging in the United States in the Twentieth Century".

The final chapter, entitled "The Transparent Body in Late Twentieth Century Culture", is fascinating, though. To quote: "The reality of seeing through and into the living body has continued to seep into cultural consciousness, transforming our sense of what can be and ought to be seen". This book may help people to appreciate and respect science by helping to show its worldwide influence on our culture and thinking.

John Mallard is professor emeritus of the Department of Bio-medical Physics and Bio-engineering, University of Aberdeen, Foresterhill, Aberdeen AB9 2ZD, UK.

\title{
Specialist books
}

\section{Excellent $\star \star \star \star \star \star$ Good $\star \star \star \star \star$ Average $\star \star \star$ Poor}

\section{Early Vertebrates}

by Philippe Janvier

Oxford University Press: 1996. Pp. 393. £75, \$135

Philippe Janvier has excelled himself with this landmark publication, which surely ranks as a modern classic. No other textbook has dealt so clearly with the origins and early evolution of vertebrates. Each chapter is lucidly written and important anatomical features are clearly illustrated, providing just the right level of technical information without getting bogged down with details. Competing evolutionary relationships are cleverly depicted on opposite sides of certain illustrations, driving home the message that many current issues are unresolved. This is an essential source book for teachers and students of vertebrate anatomy and evolution.

Anatomy lies at the core of the book, but there are also useful chapters on Recent vertebrate diversity, phylogenetic principles, the fossil record, and current theories and controversies. There are also entertaining chapters on the history of important early vertebrate discoveries and discoverers, from whom I note Janvier modestly excluded himself; that was the most serious omission I could find. John G. Maisey Department of Vertebrate Paleontology, American Museum of Natural History, New York, USA.

\begin{tabular}{|c|c|}
\hline Range & $\star \star \star \star \star$ \\
\hline Depth & 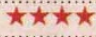 \\
\hline Accuracy & 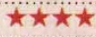 \\
\hline Up-to-dateness &  \\
\hline Accessibility & $\star \star \star \star \star$ \\
\hline Style &  \\
\hline
\end{tabular}

\section{Supersymmetry in Disorder and} Chaos

by Konstantin Efetov

Cambridge University Press:1997. Pp. 441. £65, $\$ 100$

Those strangely attracted to chaotic dynamics by the fearful symmetries of nature need read no further. Supersymmetry is a serious business, having little in common with the phenomenal fantasies that share the name.

This super-saturated manifesto from the Landau Institute is on the spectre of supermathematics of commuting and anticommuting Grassmann variables. Ground rules are provided for a game, a method of calculation that leads from random field theory to quantum chaos, for those proficient in mathematical analysis, complex variables and quantum mechanics. The rules are applicable to the theoretical, and not just imaginary, physics of disordered systems of practical importance, leading to localization and mesoscopic and quantum effects. Its power over perturbative approaches is illustrated by applications to disordered metals and semiconductors, including mesoscopic quantum devices that are emerging as the basis for nanotechnologies. Arun Holden Centre for Nonlinear Studies, University of Leeds, UK.

Range
Depth
Accuracy
Up-to-dateness
Accessibility
Style

$\star \star \star \star$
$\star \star \star \star$
$\star \star \star \star \star$
$\star \star \star$
$\star \star$
$\star \star$
$\star \star \star$

\section{Concise Thermodynamics}

by Jeremy Dunning-Davies

Albion: 1996. Pp. 126. $£ 15$

Thermodynamics is such a widely used discipline that any new book on the subject warrants attention. This pleasant and slim volume offers a rapid and simple introduction that can be used by physicists, chemists and engineers.

However, one would not expect such a small book to introduce reaction rates (useful for chemists), actual efficiencies of power plants (useful for engineers) or the entropy of mixing (useful for solid-state physicists and chemists). You get what you pay for, namely the bare bones of thermodynamics. There are problems and solutions that experts will have seen before but that will help the beginner to sort out his ideas.

For those wanting just a skeleton introduction, this book may well serve their needs. The last chapter on black holes and the concluding remarks about possible thermodynamic errors in cosmology, however, will not be acceptable to the physics community, and would be better omitted in any future edition.

Peter T.Landsberg Faculty of Mathematical Studies, University of Southampton, UK.

Range
Depth
Accuracy
Up-to-dateness
Accessibility
Style

